\title{
Neuroimaging heats up
}

New study hopes to validate imaging tools and markers for tracking Alzheimer's disease.

Mark Ratner

A big challenge facing developing drugs for Alzheimer's disease is that measurements of disease progression are mostly observational - such as the use of memory or cognitive tests - and require years of follow-up. Diagnostic imaging could reduce this time dramatically, but tools and methods are a long way from being validated. Now, initiatives are sprouting up to fill the gap.

In June, a public-private consortium known as the Alzheimer's Disease Neuroimaging Initiative (ADNI) launched an 800-patient longitudinal study across 50 clinical sites to validate structural imaging tools including MRI - measuring shape and size of the brain and hippocampus - and fluorodeoxyglucose positron emission tomography (FDG-PET) - measuring brain function or activity.

Four years in the planning, ADNI was established by the Foundation of the National Institutes of Health (FNIH), which supports the $\mathrm{NIH}$ through establishing both for-profit and notfor-profit relationships. ADNI aims to investigate technologies that have shown high potential in tracking disease progression, "but for which the literature is, frankly, confusing," says Pfizer's Peter Snyder, head of the ADNI study design group and chair of its Industry Scientific Advisory Board.

Confusion arises because different labs use different algorithms when imaging the brain, which can lead to varying results.

"It became clear there was a need to collaborate across labs and platforms to develop a Gold Standard reference library to compare techniques," says Snyder.

As almost two-thirds of the US\$60-million total budget for ADNI is being stumped up by federal funds, findings will be made publicly available, with baseline data expected by the end of the year.

The need for validated imaging tools and markers offset any industry concerns over sharing data and methods, or with conducting studies at a slower pace than companies are accustomed to. "The magnitude of getting agreement on clinical resources, diagnoses and the regulatory pathway to launch a new therapeutic made the need for developing a consensus obvious," says William Potter from Merck, which joined ADNI last year.

All told, eleven companies have signed up as donors. Pfizer is the largest industry contributor, putting up $\$ 4.5$ million over

5 years towards the project, along with access to antibodies and the processes for making them.

In the study, patients will be tracked for 2-3 years, and up to half of the subjects will contribute cerebrospinal fluid (CSF), a robust source of potential biomarkers such as tau, phospho-tau, amyloid and sulphatide.

Ownership and use of clinical samples collected in government-sponsored trials has been a dicey issue in the past. A US congressional committee is investigating whether samples collected in the NIH Intramural Research programme were either not kept track of accurately or used outside of their consented purposes.

In $\mathrm{ADNI}$, however, which is part of $\mathrm{NIH}$ extramural funding, the grantee institution is officially responsible for the samples, and data will be shared even before publication. Guidelines on access and sharing of CSF collected in the trial should be in place by the end of the year, according to Susan Molchan, ADNI project officer at the NIH's National Institute on Aging (NIA).

Alternatively, focusing on measuring one or two endpoints linked to a single drug could be a faster way of moving a programme into clinical trials. Last month, Roche and GE Healthcare, neither of whom are funding ADNI, announced a Phase I collaboration around a Roche anti-amyloid drug candidate, due to begin next year.

Patients will be monitored for drug response using a hydroxylated benzothiole PET tracer biomarker from GE to track levels of amyloid plaque in the brain. The goal of this parallel drug-diagnostic development plan is to determine whether the Roche compound can decrease the amount of plaque, and whether this makes a clinical difference.

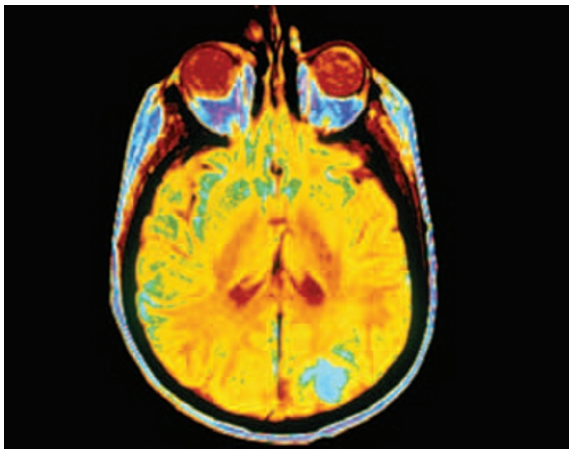

